\title{
Stacked Subwavelength Gratings as Circular Polarization Filters
}

Gregory P. Nordin

nordin@byu.edu

P. C. Deguzman

Follow this and additional works at: https://scholarsarchive.byu.edu/facpub

Part of the Electrical and Computer Engineering Commons

\section{Original Publication Citation}

P. C. Deguzman and G. P. Nordin, "Stacked Subwavelength Gratings as Circular Polarization Filters," Appl. Opt. 4(31), pp. 5731-5737 (21)

\section{BYU ScholarsArchive Citation}

Nordin, Gregory P. and Deguzman, P. C., "Stacked Subwavelength Gratings as Circular Polarization Filters" (2001). Faculty Publications. 1084.

https://scholarsarchive.byu.edu/facpub/1084 


\title{
Stacked subwavelength gratings as circular polarization filters
}

\author{
Panfilo C. Deguzman and Gregory P. Nordin
}

\begin{abstract}
We have stacked subwavelength gratings (SWGs) on a single substrate to create a compact, integrated circular polarization filter. The SWGs consist of a wire grid polarizer and a broadband form-birefringent quarter-wave plate (QWP). Rigorous coupled-wave analysis was used to design the QWP for operation over the 3.5-5.0- $\mu \mathrm{m}$ wavelength range. The fabricated silicon broadband QWP exhibited a phase retardance of $82-97^{\circ}$ across this wavelength range. Two stacked structures are presented, each with a different wire grid polarizer fabricated on an organic planarization layer (SU-8) that is deposited on a QWP grating. Transmittance measurements of the first structure when illuminated with nominally right- and left-circularly polarized light indicate a circular extinction ratio (CER) limited by the low linear extinction ratio of the polarizer. Use of a wire grid polarizer with a higher extinct ratio led to a stacked SWG structure that demonstrated CERs of 10-45 across the 3.5-5.0- $\mu \mathrm{m}$ wavelength range. (C) 2001 Optical Society of America
\end{abstract}

OCIS codes: $230.5440,050.1950,260.1440,260.5430$.

\section{Introduction}

Subwavelength grating (SWG) structures have been demonstrated in many device configurations such as polarization components, ${ }^{1,2}$ antireflection (AR) surfaces, ${ }^{3,4}$ narrow-band filters,, 6 and graded-index components. ${ }^{7,8}$ (For purposes of this paper, a SWG is defined as one that gives rise to propagation of only the zero-reflected and zero-transmitted diffraction orders for the desired application.) An attractive option that has been previously unexplored to our knowledge is the stacking of multiple SWGs into a single compact device. For some applications this can be analogous to a sequence of discrete bulk components being reduced to a stack of thin SWG layers (possibly interleaved with homogeneous layers) on a common substrate. This approach offers several potential advantages: significant size reduction compared with a sequence of otherwise discrete components, precise and stable alignment between components, flexibility in the design of complex optical systems, and possible low-cost fabrication. In

The authors are with the Department of Electrical and Computer Engineering, University of Alabama in Huntsville, Huntsville, Alabama 35899. G. P. Nordin's e-mail address is nordin@ece.uah.edu.

Received 5 September 2000; revised manuscript received 8 June 2001.

0003-6935/01/315731-07\$15.00/0

(C) 2001 Optical Society of America addition, arrays of small-aperture, stacked SWGs can be formed to provide a large number of parallel optical channels. ${ }^{9}$

As an example of stacked SWGs, we present the implementation of a broadband circular polarization filter. Applications of such filters include imaging polarimetry ${ }^{10}$ and optical isolators. The SWG stack that we describe in this paper consists of a wire grid polarizer that is fabricated on top of a broadband form-birefringent quarter-wave plate (QWP). The QWP is designed for operation over the wavelength range of 3.5-5.0 $\mu \mathrm{m}$. An organic layer is used between the QWP and the wire grid polarizer to planarize the QWP grating's surface relief. Design of the SWG layers is based on rigorous coupled-wave analysis (RCWA). ${ }^{11}$

In the following sections we describe the development of two SWG stacks, each of which acts as a circular polarization filter. In Section 2 we first discuss the broadband form-birefringent QWP. Then in Section 3 we describe the fabrication and performance of a stack that includes a low extinction ratio polarizer. We refer to this as stack 1. In Section 4 we examine a stack that has a polarizer with a significantly larger extinction ratio, which we refer to as stack 2. A summary is provided in Section 5.

\section{Broadband Form-Birefringent Quarter-Wave Plate}

Form-birefringent wave plates for narrow-band operation have been implemented as subwavelength surface relief gratings in silicon nitride, ${ }^{12} \mathrm{GaAs},{ }^{13}$ and 


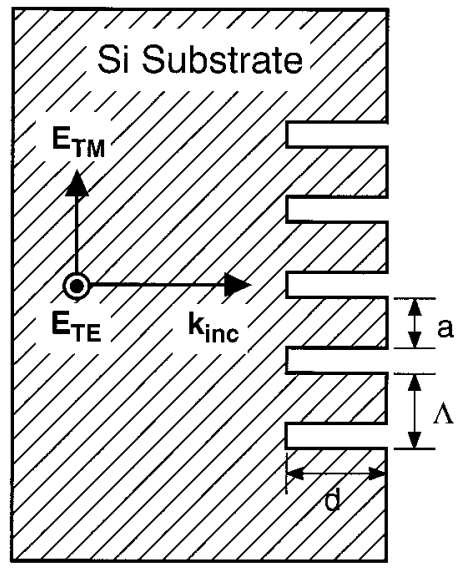

Fig. 1. Schematic diagram of a form-birefringent wave plate implemented as a subwavelength surface relief grating. Orientations of the TE and TM electric field vectors are shown for a normally incident beam.

amorphous silicon. ${ }^{14}$ For broadband operation, Kikuta et al. recently proposed a method for creating achromatic form-birefringent wave plates. ${ }^{15}$ When the grating period is of the order of the optical wavelength, the strong dispersion of the effective refractive indices can be used to compensate for the usual $1 / \lambda$ dependence of the phase retardation. We previously reported the implementation of a broadband wave plate that makes use of form-birefringencebased dispersion using a SWG in silicon operating in an ambient medium of air. ${ }^{16}$ In this section we extend this structure to the case of a Si SWG in which the grating surface relief is planarized by a low-index thin film. In our case we use an epoxy-based, negative resist, SU-8 (MicroChem Corp., Newton, Mass.).

\section{A. Design}

A schematic illustration of a form-birefringent SWG is shown in Fig. 1. Our design goal for the QWP was a retardance $\Gamma$ of $0.5 \pi \pm 0.1 \pi$ over the $3.5-5.0-\mu \mathrm{m}$ wavelength range. An additional requirement was that the design be as insensitive as possible to fabrication errors, which primarily involve the grating etch depth and fill factor (i.e., ratio of the grating ridge width $a$ and the grating period $\Lambda$ ). Of these, the fill factor is typically the most challenging to control and imposes the tightest fabrication constraints.

The design process involved the systematic variation of the grating period, fill factor, and etch depth to find a set of parameters that would meet the design criteria. RCWA ${ }^{11}$ was used to calculate the retardance of the grating and the transmittance of TE and TM linearly polarized light normally incident on the grating through the substrate. A Si substrate is assumed with dispersion as indicated in Ref. 17. The grating grooves and the ambient medium are assumed to be SU-8. The optical constants of SU-8 were obtained through ellipsometric measurements by the J. A. Woollam Co. (Lincoln, Neb.) for a sample prepared in our laboratory.

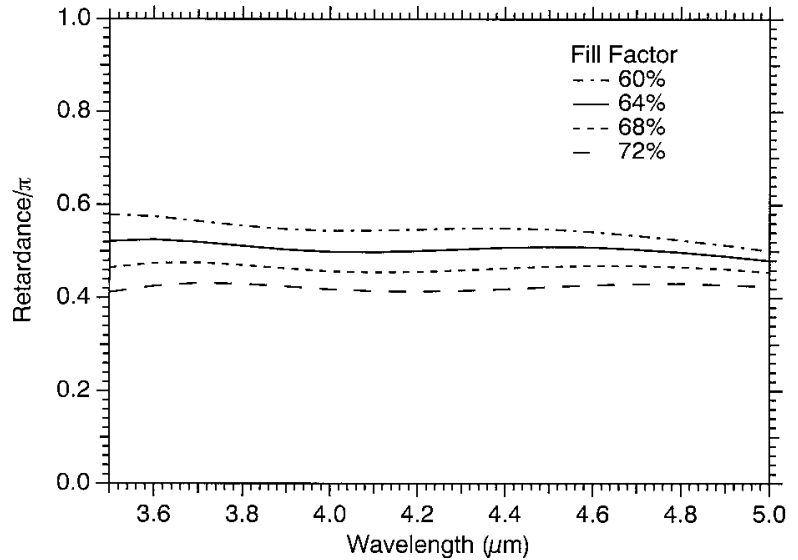

Fig. 2. RCWA simulation results for phase retardation normalized by $\pi$ for a Si QWP $(\Lambda=1.0 \mu \mathrm{m}, d=2.15 \mu \mathrm{m})$ in an ambient medium of SU-8 as a function of wavelength parameterized by the grating fill factor $(a / \Lambda)$.

We found that a SWG with a $1-\mu \mathrm{m}$ period, $64 \%$ fill factor, and $2.15-\mu \mathrm{m}$ etch depth best met our design criteria. As shown in the RCWA simulation results in Fig. 2, this design yields a retardance of $0.48 \pi$ to $0.52 \pi\left(90^{\circ} \pm 3.6^{\circ}\right)$ over the $3.5-5.0-\mu \mathrm{m}$ wavelength range. Moreover, fill factors of $60-72 \%$ satisfy our retardance requirement, which allows for a reasonable variability in process parameters.

\section{B. Fabrication}

We fabricated our broadband QWP design on the surface of 75-mm-diameter $p$-type $\langle 100\rangle \mathrm{Si}$ substrates with a resistivity of $1-20 \Omega-\mathrm{cm}$. The photolithography was performed in a contact mask aligner (custom model from AB-Manufacturing, San Jose, Calif.) with a 5 in. $(13 \mathrm{~cm}) \times 5$ in. $(13 \mathrm{~cm})$ dark-field photomask in which the central $1.3 \mathrm{~cm} \times 1.3 \mathrm{~cm}$ is comprised of 500 -nm-wide open lines in the chrome. These lines are placed on $1-\mu \mathrm{m}$ centers. Anisotropic etching of the Si substrate is performed in a PlasmaTherm 790 reactive ion etcher with 9-in.- (23-cm-) diameter electrodes. A graphite cathode was used for all the etches described below.

We prepared the Si wafers for photolithography by first applying an optically absorptive antireflective coating (ARC) from Brewer Science. The purpose of this layer is to reduce standing waves in the photoresist that are due to interference from light reflected by the substrate. Following the ARC, a $500-\mathrm{nm}$ layer of Shipley 1805 photoresist is spin coated and then soft baked at $115^{\circ} \mathrm{C}$ for $1 \mathrm{~min}$ on a vacuum hot plate. The resist is exposed in the contact mask aligner and immersed in Microposit 352 developer to produce a photoresist grating with an approximately $20 \%$ fill factor. An anisotropic etch in the reactive ion etcher by use of helium and oxygen [gas flow rates of 8 SCCM (SCCM denotes cubic centimeters per minute at STP) each, 175 -W rf power, 10 -mTorr pressure, and 3-min etch time] transfers the resist pattern into the ARC layer. We then patterned a $\mathrm{Cr}$ etch mask on the Si substrate using a lift-off process ${ }^{18}$ 


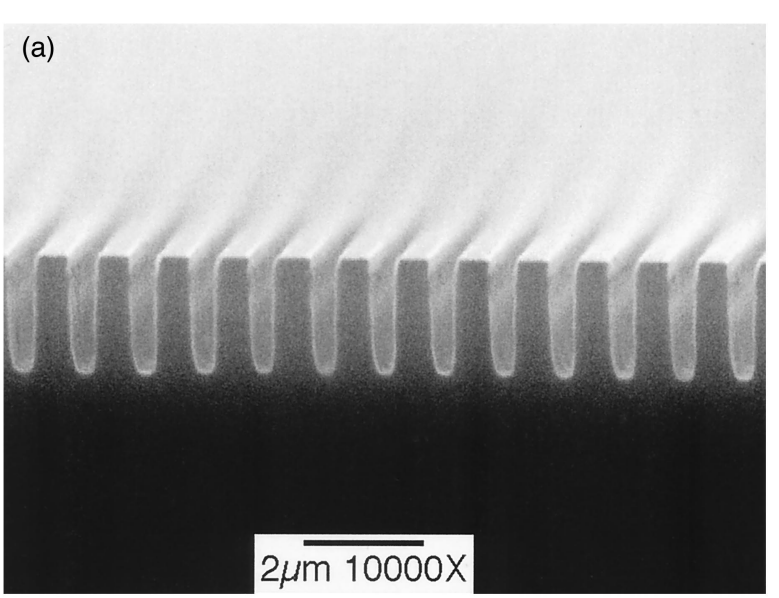

(b)

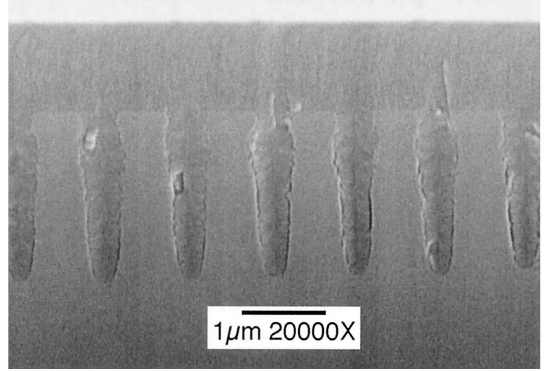

Fig. 3. SEM cross-sectional image of (a) etched Si grating and (b) grating planarized with SU-8.

in which a 250 -nm-thick chrome layer is thermally evaporated followed by immersion in Microposit 1165 remover to strip the resist and ARC layers, thereby leaving behind a $\mathrm{Cr}$ grating pattern on the $\mathrm{Si}$.

The Cr grating pattern is transferred into the $\mathrm{Si}$ substrate by a two-step etch process that relies on the formation of polymer-based sidewall passivation to achieve an anisotropic etch. ${ }^{19}$ The first etch step uses $\mathrm{CHF}_{3}$ and $\mathrm{SF}_{6}$ (35- and 5-SCCM gas flow rates) and the second uses just $\mathrm{CF}_{4}$ (40 SCCM). In both cases the rf power is $125 \mathrm{~W}$ and the chamber pressure is 10 mTorr. We typically etch three wafers simultaneously for a total of $165 \mathrm{~min}$.

A representative grating structure fabricated by this procedure is shown in Fig. 3(a). A grating planarized with SU-8 is shown in Fig. 3(b). The grating in Fig. 3(a) has a 2- $\mu \mathrm{m}$ etch depth, a slightly undercut profile at the top of each groove, and somewhat sloped sidewalls. The 1.1- $\mu$ m-thick SU-8 layer shown in Fig. 3(b) is clearly sufficient to planarize the grating's surface relief.

\section{Experimental Measurement}

A Fourier-transform infrared- (FTIR-) based spectropolarimeter ${ }^{20}$ was used to measure the retardance of the sample shown in Fig. 3(b), and a FTIR spectrometer (Bio-Rad FTS60A) was used to measure the transmittance for normally incident TE and TM illumination. For this particular sample, the back side of the $\mathrm{Si}$ wafer was not $\mathrm{AR}$ coated, which results in a $30 \%$ Fresnel reflection loss at this surface. The
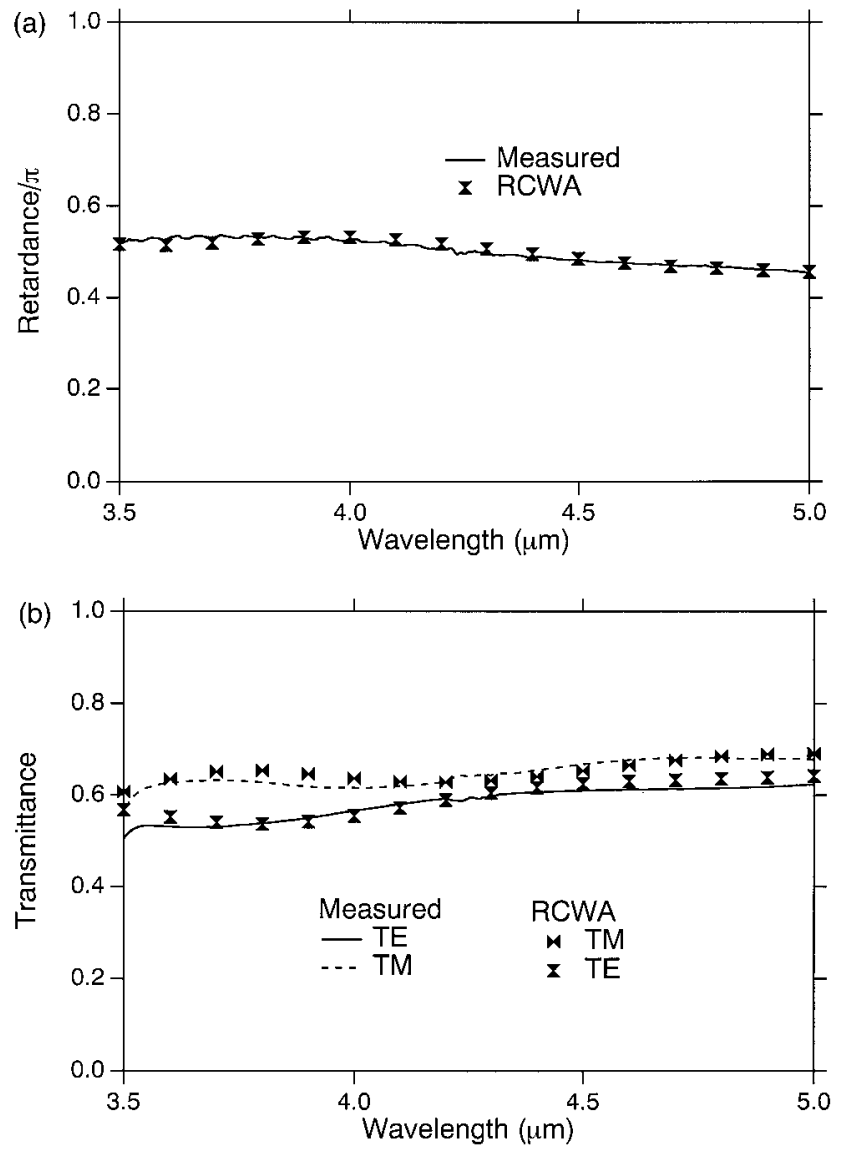

Fig. 4. (a) Measured and simulated retardance as a function of wavelength and (b) corresponding TE and TM transmittances.

RCWA simulation results presented below are corrected by this factor.

Figure 4(a) shows the retardance as a function of wavelength. A retardance of $0.45 \pi$ to $0.53 \pi$ (82$97^{\circ}$ ) was measured for this sample, which is well within our requirements. The external TE and TM transmittances are shown in Fig. 4(b). These vary from $53 \%$ to $68 \%$, and after we account for multiple reflections within the substrate, this implies a transmittance through the SU-8 coated surface of 69-96\%.

For comparison, RCWA was used to simulate the performance of the QWP in Fig. 3. The etched grating profile was approximated by a series of binary grating layers, the parameters for which are listed in Table 1. As illustrated in Fig. 4, the agreement be-

Table 1. RCWA Parameters for a Si Grating with SU-8 Filling the Grooves and a 1.1- $\mu$ m-Thick Homogeneous SU-8 Film on Top ${ }^{a}$

\begin{tabular}{ccc}
\hline Layer & Thickness $(\mu \mathrm{m})$ & Si Ridge Width $(\mu \mathrm{m})$ \\
\hline 1 & 0.174 & 0.769 \\
2 & 0.363 & 0.668 \\
3 & 0.363 & 0.618 \\
4 & 0.363 & 0.569 \\
5 & 0.363 & 0.541 \\
6 & 0.363 & 0.569 \\
\hline
\end{tabular}

${ }^{a}$ The entrance material is $\mathrm{Si}$, and the exit material is air. 
tween simulation and measurement is excellent for both the retardance and the TE and TM transmittance. Further simulation studies show that the retardance is nearly unaffected by the thickness of the homogeneous SU-8 layer above the grating, whereas the usual periodic variation of transmittance as a function of thickness for a given wavelength that is typical of thin-film interference effects is exhibited.

\section{Circular Polarization Filter: Stack 1}

In this section we discuss the implementation of a circular polarization filter consisting of a wire grid polarizer fabricated on top of the QWP described above. The metal ridges of the wire grid polarizer are rotated counterclockwise $45^{\circ}$ with respect to the Si ridges of the QWP when viewed from the polarizer side of the substrate. Because the effective refractive index of the QWP for TM illumination is less than the effective index for TE illumination, the structure should pass light that is left-circularly polarized (LCP) and reject (i.e., reflect) right-circularly polarized (RCP) light. Here we define RCP as circular polarization with counterclockwise rotation of the electric field vector when viewed by an observer toward whom the light propagates and vice versa for LCP.

\section{A. Wire Grid Polarizer}

Before fabricating a wire grid polarizer on a QWP, we first developed our fabrication processes and validated our polarizer design by fabricating wire grid polarizers on bare Si substrates. We began by sputtering uniform films of 160-nm-thick Mo on 100-nmthick $\mathrm{SiO}_{2}$. The $\mathrm{SiO}_{2}$ is used as an etch stop for the Mo etch. We then spin coated $220 \mathrm{~nm}$ of ARC, followed by $500 \mathrm{~nm}$ of Shipley 1805 photoresist (processed as described above for the QWP fabrication). A $0.5-\mu \mathrm{m}$ period grating is patterned in the photoresist with interference lithography as described in an earlier paper, ${ }^{10}$ followed by an anisotropic ARC etch to transfer the pattern into the ARC. Next, $25 \mathrm{~nm}$ of $\mathrm{Cr}$ is thermally evaporated onto the grating and lifted off to leave behind a $0.5-\mu \mathrm{m}$ period $\mathrm{Cr}$ etch mask on the Mo.

There are two stages to the ensuing Mo reactive ion etch. In the first, $\mathrm{SF}_{6}$ (40-SCCM flow rate, 15 -mTorr chamber pressure, $100-\mathrm{W}$ rf power, and 5.5-min etch time) is used to etch through $95 \%$ of the Mo film. In the second, a $\mathrm{SF}_{6} / \mathrm{Ar}$ gas chemistry (30/10 SCCM, 15-mTorr chamber pressure, $400-\mathrm{W}$ rf power, and 30 -s etch time) is used to break through the final portion of the Mo film into the $\mathrm{SiO}_{2}$ etch stop layer. A typical result is shown in Fig. 5. It is evident that there is a small amount of undercutting below the thin $\mathrm{Cr}$ etch mask because the $\mathrm{SF}_{6}$ etch is not strictly anisotropic, whereas the etch terminates, as desired, in the $\mathrm{SiO}_{2}$ layer.

The polarization performance of a fabricated sample was measured in the FTIR spectrometer mentioned above. A commercially available wire grid polarizer (from Graseby Specac) was used to generate the incident TM and TE polarization states. It has

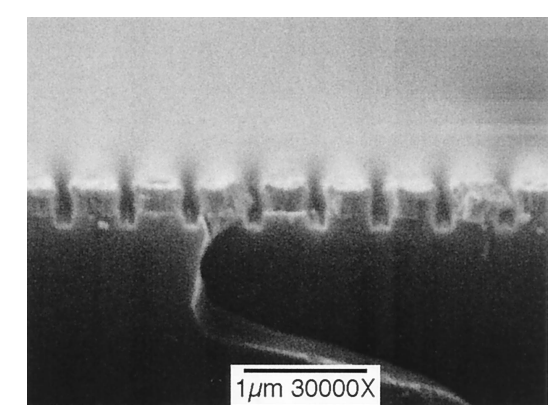

Fig. 5. SEM cross-sectional image of a wire grid polarizer sample.

an extinction ratio of approximately $130-190$ in the 3.5-5.0- $\mu \mathrm{m}$ wavelength range. We define extinction ratio as the ratio of the polarizer transmittance for normally incident TM and TE linearly polarized light $\left(T_{\mathrm{TM}} / T_{\mathrm{TE}}\right)$, with TM and TE as defined in Fig. 1 except with metal ridges replacing the Si ridges.

Measured external transmittances for TM and TE illumination as a function of wavelength are shown in Fig. 6. RCWA simulation results are in good agreement with the measurement when corrected for Fresnel reflection loss at the non-AR-coated back surface of the substrate. Ellipsometrically determined optical constants for sputtered $\mathrm{Mo}$ and $\mathrm{SiO}_{2}$ (measured by the J. A. Woollam Co.) were used in the simulation. From the measured TM and TE transmittances, and when we account for the finite extinction ratio of the reference polarizer, the extinction ratio for the polarizer of Fig. 6 varies from approximately $41-47$ over the $3.5-5.0-\mu \mathrm{m}$ wavelength range. When we correct for the Fresnel reflection loss at the back surface of the substrate, approximately $80 \%$ of the TM illumination is transmitted through the polarizer side of the sample.

\section{B. Stack 1 Fabrication}

The stack 1 circular polarization filter consists of the $0.5-\mu \mathrm{m}$ period wire grid polarizer described in Subsection 3.A fabricated directly on top of the SU-8

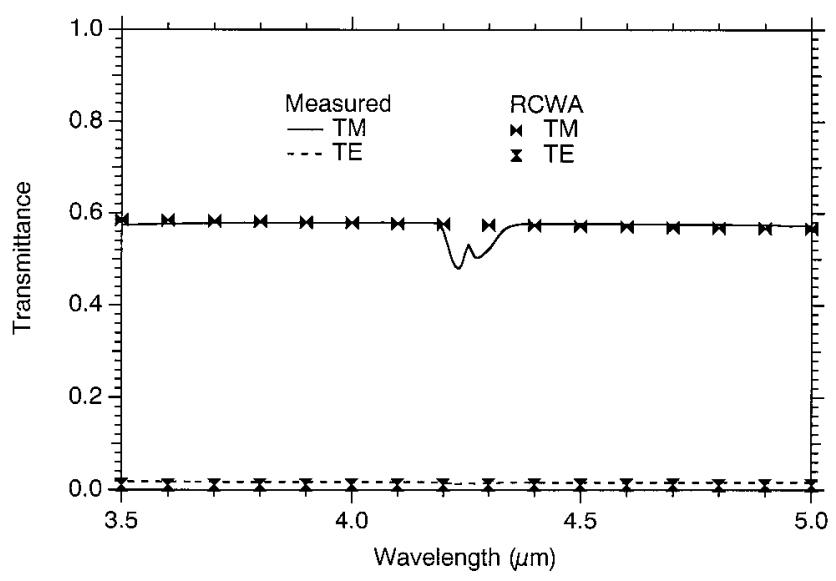

Fig. 6. Transmittance measurements of TM and TE illumination through the polarizer in Fig. 5 with corresponding RCWA results. The dip in the center of the measured TM curve is due to $\mathrm{CO}_{2}$ absorption in the FTIR. 


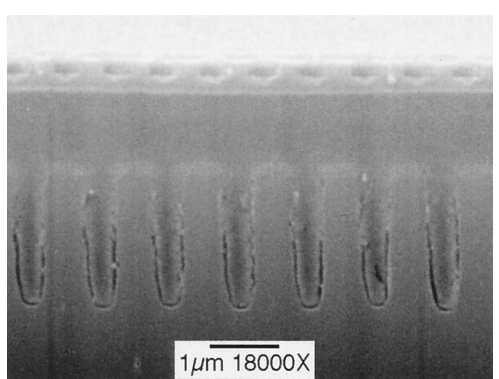

Fig. 7. SEM cross-sectional image of the stack 1 circular polarizer. See text for details.

planarization layer of the form-birefringent QWP described in Section 2. The polarizer fabrication process is the same as above, including the $\mathrm{SiO}_{2}$ etch stop layer.

A scanning electron microscope (SEM) image cross section of a stack 1 sample is shown in Fig. 7. The $\mathrm{Si}$ ridges are oriented normal to the plane of the image, whereas the metal ridges of the wire grid polarizer are oriented $45 \mathrm{deg}$ out of this plane, thereby rendering them somewhat difficult to see clearly. Detailed observation of these ridges shows that their fill factor is only $35 \%$. This is significantly smaller than the wire grid polarizer of Fig. 5 and is due to a difference in the interference lithography exposure dosage. Because the wire grid polarizer extinction ratio is strongly dependent on the metal fill factor, we expect a much lower extinction ratio for the wire grid polarizer in Fig. 7.

To confirm this we measured the extinction ratio directly by illuminating the polarizer side of the stack 1 sample and followed the same procedure as in Subsection 3.A. The measured extinction ratio varies from 10 to 15 over the 3.5-5.0- $\mu \mathrm{m}$ wavelength range. As is easily shown (see, for example, Ref. 21), the circular extinction ratio (CER) (i.e., ratio of the transmittance of the stack for LCP and RCP incident illumination $T_{\mathrm{LCP}} / T_{\mathrm{RCP}}$ ) of a circular polarization filter must be less than or equal to the linear extinction ratio of the polarizer. We therefore expect the CER for stack 1 not to exceed the range of 10-15.

\section{Experimental Measurement}

We tested the polarization performance of the stack 1 sample with the FTIR spectrometer using the test setup schematically illustrated in Fig. 8. Bulk po-

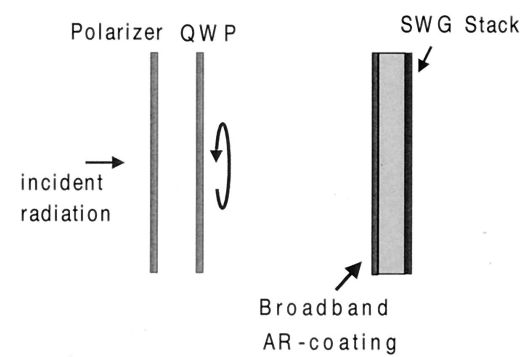

Fig. 8. Schematic illustration of circular polarization filter test geometry in the FTIR spectrometer.

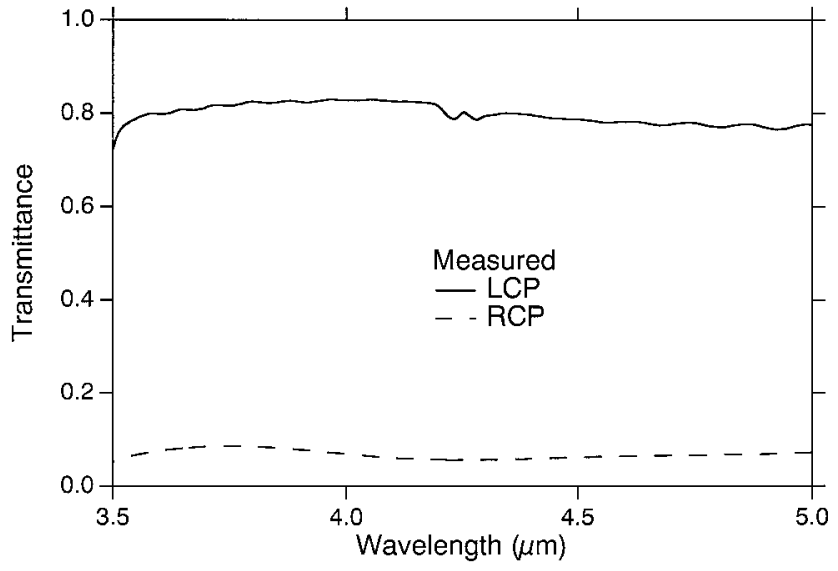

Fig. 9. Measured transmittance of the stack 1 sample with broadband ARC for normally incident LCP and RCP illumination.

larization components (consisting of a formbirefringent QWP fabricated in our laboratory and the Graseby Specac wire grid polarizer) generate the incident LCP and RCP illuminations that first pass through the AR-coated back surface of the substrate and then into the QWP polarizer stack. Because the bulk components are nonideal, the actual polarization state incident on the sample is somewhat elliptical in both cases. We measured the performance of each bulk component, so we are able to calculate the incident polarization state as a function of wavelength. The measured stack 1 sample had a commercial broadband ARC for the $3-5.0-\mu \mathrm{m}$ wavelength range applied to the back side of the Si substrate to suppress Fresnel reflection losses.

The transmittance measurements for nominal LCP and RCP normally incident illumination for the stack 1 sample are shown in Fig. 9. (As shown in Fig. 8, the QWP side of the stack is illuminated through the substrate.) The stack acts as a circular polarization filter in that approximately $80 \%$ of the incident LCP illumination is transmitted compared with less than $7 \%$ of the RCP illumination. The ratio between the measured curves varies from 9 to 13 across the 3.5$5.0-\mu \mathrm{m}$ wavelength range, which is comparable to the linear extinction ratio of the wire grid polarizer. We therefore conclude that the CER performance of this stack is limited by the low wire grid polarizer extinction ratio.

\section{Circular Polarization Filter: Stack 2}

A second stack (referred to as stack 2) was fabricated in which we used a higher extinction ratio wire grid polarizer to verify that a larger CER can be achieved than was observed for stack 1 . The wire grid polarizer for stack 2 has a $1.0-\mu \mathrm{m}$ period grating composed of 160-nm-thick Mo and 300-nm-thick Cr. As seen below, the increased metal thickness of the wires more than compensates for the larger grating period to yield a significantly improved extinction ratio compared with the polarizer of stack 1 .

The fabrication of the improved polarizer on a planarized QWP proceeds as follows: (1) sputter a 


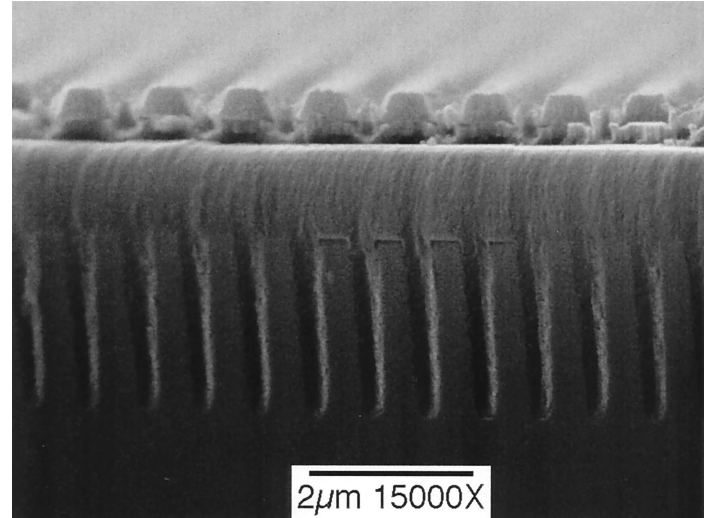

Fig. 10. SEM cross-sectional image of a stack 2 circular polarizer. See text for details.

100-nm $\mathrm{SiO}_{2}$ etch stop layer; (2) sputter a $160-\mathrm{nm}$ Mo layer; (3) deposit the ARC and photoresist as outlined in Subsection 3.A; (4) expose the photoresist with a $1.0-\mu \mathrm{m}$ period mask in a contact mask aligner and develop as described in Subsection 2.B; (5) etch the ARC and evaporate the $\mathrm{Cr}$, again as described in Subsection 2.B, except with 300-nm-thick Cr; (6) lift off the Cr; and (7) etch the Mo as described in Subsection 3.A.

A cross-sectional SEM image of the stack 2 sample is shown in Fig. 10. Note that in this case the orientation of the wire grid ridges is nearly perpendicular to the plane of the image, whereas the QWP ridges are at an angle and therefore appear distorted. For this sample, the SU-8 layer is $1.3 \mu \mathrm{m}$ thick and the QWP is $2.1 \mu \mathrm{m}$ deep.

We measured the TE and TM transmittance of the wire grid polarizer using the same technique as for the wire grid polarizer of stack 1 . The result is shown in Fig. 11. When we account for the finite extinction ratio of the Graseby Specac polarizer used to generate the incident polarization states for the measurements, a lower bound for the extinction ratio of the polarizer of stack 2 is 210 in the $3.5-5.0-\mu \mathrm{m}$

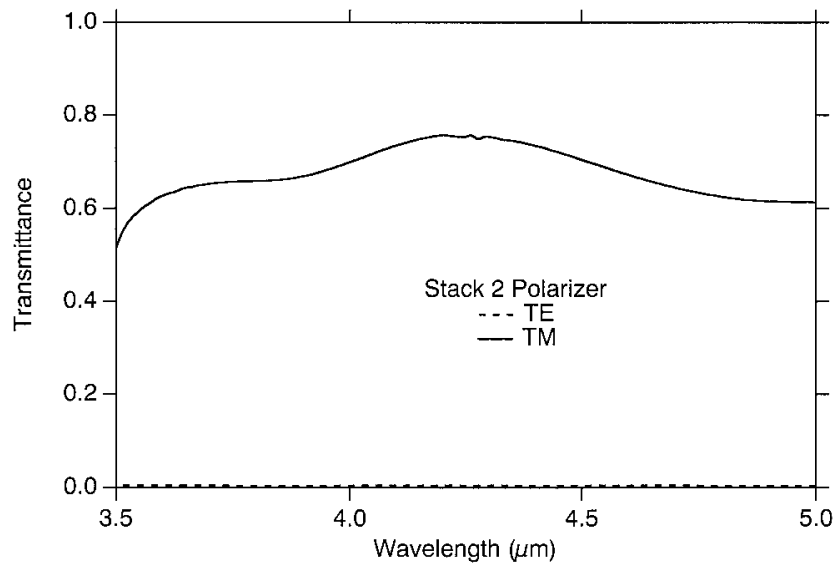

Fig. 11. Measured transmittance of stack 2 illuminated from the polarizer side of the sample for linearly polarized TE and TM illumination.

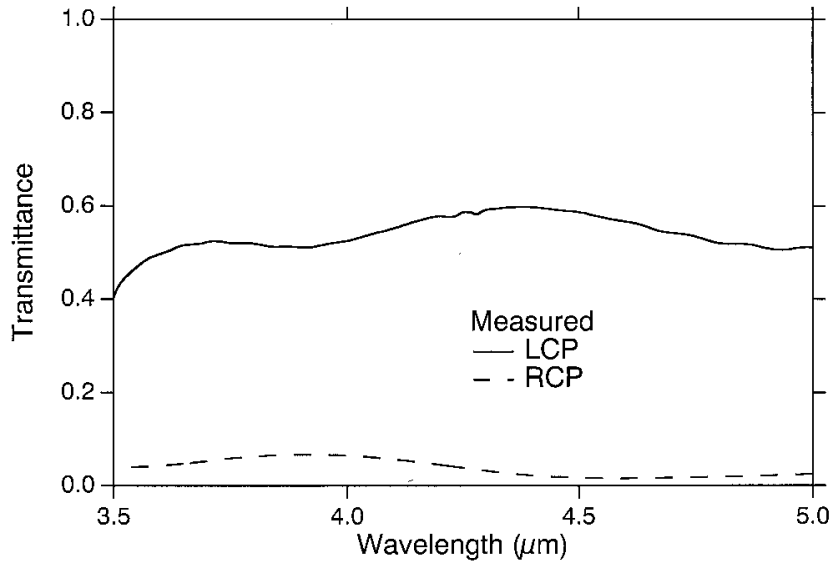

Fig. 12. Measured transmittance of stack 2 sample illuminated through the back side of the sample with LCP and RCP light.

wavelength region. This is over an order of magnitude better than the polarizer in stack 1 .

The stack 2 sample had the same broadband ARC applied to the back side of the wafer as for stack 1 . We measured the transmittance of stack 2 when illuminated through the substrate for nominally LCP and RCP incident light using the same method as outlined in Subsection 3.C. The result is shown in Fig. 12. Between $40 \%$ and $60 \%$ of the LCP illumination is transmitted through the stack. This is reduced compared with stack 1 primarily because of the lower TM transmittance of the stack 2 polarizer. The transmission of the RCP illumination varies between $1.4 \%$ and $6.5 \%$.

The CER for stack 2 ranges between 10 and 45 over the 3.5-5.0- $\mu \mathrm{m}$ wavelength range. Given its relatively high extinction ratio, the polarizer performance is clearly not a limiting factor in the CER. Instead, there are several other potential limitations. The first is nonideal QWP behavior, which results from a phase retardation different from $\pi / 2$ and nonequal TE and TM transmittances through the QWP. Second, multiple reflections between the polarizer and the QWP can lead to leakage of RCP light through the stack. And third, the incident LCP and RCP states are not ideal and ultimately limit the measured CER. The determination of the relative effects of these factors is a topic for future research.

\section{Summary}

We have demonstrated the use of stacked SWGs as circular polarization filters. Filters with two different wire grid polarizers were fabricated. The first had a CER limited by the extinction ratio of the wire grid polarizer. The second was fabricated with a higher extinction ratio polarizer and demonstrated a CER that varied between 10 and 45 over the 3.5$5.0-\mu \mathrm{m}$ wavelength range.

Our future research includes improving the CER by addressing the issues outlined above, examining use of SWG circular polarization filters in a variety of applications, analyzing other stacked SWG configurations, and applying the SWG circular polarization 
filters to other wavelength ranges. In particular, our experience indicates that a circular polarization filter should be achievable for the 1.5- $\mu \mathrm{m}$ wavelength range if the QWP and wire grid polarizer grating periods are suitably reduced (and of course the QWP fill factor and etch depth will need to be redesigned as well). If just the $C$ and $L$ bands are to be covered, the broadband requirement for the QWP is significantly relaxed over the case considered in this paper, which helps simplify the QWP design. We anticipate that the primary challenge to realize a circular polarization filter with high CER over the $C$ and $L$ bands is likely to be the achievement of a tight tolerance on the QWP fill factor.

G. P. Nordin acknowledges support by National Science Foundation CAREER award ECS-9625040 and grant EPS-9720653. P. Deguzman acknowledges support by a National Science Foundation traineeship (grant GER-9553475).

\section{References}

1. R. C. Enger and S. K. Case, "Optical elements with ultrahigh spatial-frequency surface corrugations," Appl. Opt. 22, 3220 3228 (1983).

2. G. R. Bird and M. Parrish, Jr., "The wire grid as a nearinfrared polarizer," J. Opt. Soc. Am. 50, 886-891 (1960).

3. E. B. Grann, M. G. Moharam, and D. A. Pommet, "Optimal design for antireflective tapered two-dimensional subwavelength grating structures,” J. Vac. Sci. Technol. B 12, 333-339 (1995).

4. D. H. Raguin and G. M. Morris, "Antireflection structured surfaces for the infrared spectral region,” Appl. Opt. 32, 11541167 (1993).

5. R. Magnusson and S. S. Wang, "New principle for optical filters," Appl. Phys. Lett. 61, 1022-1024 (1992).

6. S. M. Norton, T. Erdogan, and G. M. Morris, "Coupled-mode theory of resonant-grating filters," J. Opt. Soc. Am. A 14, 629 639 (1997).

7. W. Stork, N. Streibl, H. Haidner, and P. Kipfer, "Artificial distributed-index media fabricated by zero-order gratings," Opt. Lett. 16, 1921-1923 (1991).

8. J. R. Wendt, G. A. Vawter, R. E. Smith, and M. E. Warren,
"Subwavelength, binary lenses at infrared wavelengths," J. Vac. Sci. Technol. B 15, 2946-2949 (1997).

9. G. P. Nordin, J. T. Meier, P. C. Deguzman, and M. W. Jones, "Diffractive optical element for Stokes vector measurement with a focal plane array," in Polarization: Measurement, Analysis, and Remote Sensing II, D. H. Goldstein and D. B. Chenault, eds., Proc. SPIE 3754, 169-177 (1999).

10. G. P. Nordin, J. T. Meier, P. C. Deguzman, and M. W. Jones, "Micropolarizer array for infrared imaging polarimetry," J. Opt. Soc. Am. A 16, 1168-1174 (1999).

11. M. G. Moharam, E. B. Grann, D. A. Pommet, and T. K. Gaylord, "Stable implementation of the rigorous coupled-wave analysis for surface relief gratings: enhanced transmittance matrix approach,” J. Opt. Soc. Am. A 12, 1077-1086 (1995).

12. D. C. Flanders, "Submicrometer periodicity gratings as artificial anisotropic dielectrics," Appl. Phys. Lett. 42, 492-494 (1983).

13. A. G. Lopez and H. G. Craighead, "Wave-plate polarizing beam splitter based on a form-birefringent multilayer grating," Opt. Lett. 23, 1627-1629 (1998).

14. S. Y. Chou, S. J. Schablitsky, and L. Zhuang, "Application of amorphous silicon gratings in polarization switching verticalcavity surface-emitting lasers," J. Vac. Sci. Technol. B 15, 2864-2867 (1997).

15. H. Kikuta, Y. Ohira, and K. Iwata, "Achromatic quarter-wave plates using the dispersion of form birefringence," Appl. Opt. 36, 1566-1572 (1997).

16. G. P. Nordin and P. C. Deguzman, "Broadband form birefringent quarter-wave plate for the mid-infrared wavelength region," Opt. Express 5, 163-168 (1999), http://www. opticsexpress.org.

17. W. L. Wolfe and G. J. Zissis, The Infrared Handbook (Infrared Information Analysis Center, Environmental Research Institute of Michigan, Ann Arbor, Mich., 1985), pp. 7-76.

18. P. Van Zant, Microchip Fabrication: A Practical Guide to Semiconductor Processing, 3rd ed. (McGraw-Hill, New York, 1997), p. 306.

19. S. Grigoropoulos, E. Gogolides, A. D. Tserepi, and A. G. Nassiopoulos, "Highly anisotropic silicon reactive ion etching for nanofabrication using mixtures of SF6/CHF3 gases," J. Vac. Sci. Technol. B 15, 640-645 (1997).

20. D. H. Goldstein, R. A. Chipman, and D. B. Chenault, "Infrared spectropolarimetry," Opt. Eng. 28(2), 120-125 (1989).

21. P. C. Deguzman, "Stacked subwavelength gratings for imaging polarimetry," Ph.D. dissertation (University of Alabama in Huntsville, Huntsville, Ala., 2000). 